APUNTE

\title{
Antecedentes iniciales para la utilización de especies de Salix como biomasa para energía en la Región de Aysén
}

\author{
Pinilla, Juan Carlos $^{1 *}$; Riquelme, Francisca²; Acuña, Bernardo². \\ ${ }^{1}$ Instituto Forestal, Sede Biobío, Chile. jpinilla@infor.cl \\ 2 Instituto Forestal, Sede Coyhaique, Chile. \\ *Autor de correspondencia
}

DOI: https://doi.org/10.52904/0718-4646.2021.557

Recibido: 10.12.2021; Aceptado 22.12.2021

\begin{abstract}
RESUMEN
La utilización eficiente de la biomasa forestal en procesos de generación de energía es una de las líneas de trabajo del Instituto Forestal, caracterizando especies adaptadas a distintas situaciones de crecimiento y de una productividad que permitan su utilización en la generación de energía. Esto es particularmente importante para la comuna de Coyhaique, debido a la dependencia de la leña como fuente de calefacción y a la creciente necesidad de abastecimiento de biomasa para energía.

Se evaluó plantaciones experimentales de Salix alba y $S$. cinerea establecidos en la Región de Aysén, obteniendo antecedentes de crecimiento y biomasa, caracterización energética y de análisis elemental. Los resultados generales indican que, a los ocho años, $S$. alba desarrolló un mayor crecimiento en variables del árbol como en biomasa aérea, con un DAP medio de $4,7 \mathrm{~cm}$ y altura de $6,7 \mathrm{~m}$, mientras que $S$. cinérea registró valores de $2,8 \mathrm{~cm}$ y $5,5 \mathrm{~m}$ para DAP y altura, respectivamente. En biomasa aérea verde, $S$. alba presentó un valor de $18 \mathrm{~kg}$ por árbol, mientras que $S$. cinérea registró 10,4 $\mathrm{kg}$, con un valor de $41 \%$ para el peso seco en relación al peso verde de la biomasa. El Poder Calorífico Inferior fue de 18.009 y $18.163 \mathrm{~J} / \mathrm{g}$ para $S$. alba y $S$. cinérea, respectivamente, y los valores del Análisis Elemental indican que es posible su utilización como materia prima para energía.
\end{abstract}

Se requiere de ampliar estos estudios en Coyhaique y contribuir a la oferta de biomasa para su uso en energía.

Palabras clave: Salix, Energía, Crecimiento, Diversificación, Biomasa

\section{SUMMARY}

The efficient use of forest biomass in energy generation processes is one of the Forestry Institute's lines of work, characterizing species adapted to different growth situations and a productivity that allows its use in energy generation. This is particularly important for the commune of Coyhaique, due to the dependence on firewood as a source of heating and the growing need to supply biomass for energy.

Therefore, Salix alba and Salix cinerea established in the Aysén Region were evaluated, obtaining growth and biomass antecedents, energy characterization and elemental analysis. The general results indicate that, at eight years, Salix alba developed greater growth in tree variables such as aerial biomass, with a mean DBH of $4.7 \mathrm{~cm}$ and height of $6.7 \mathrm{~m}$, while Salix cinérea registered values $2.8 \mathrm{~cm}$ and $5.5 \mathrm{~m}$ for DBH and height, respectively. In green aerial biomass, Salix alba presented a value of $18 \mathrm{~kg}$ per tree, while Salix cinérea registered $10.4 \mathrm{~kg}$, with a value of $41 \%$ for the dry weight in relation to the green weight of the biomass. The Lower Calorific Power was 18,009 and 18,163 J/g for Salix alba and Salix cinérea, respectively, and the Elemental Analysis values indicate that it is possible to use it as raw material for energy.

It is necessary to expand these studies in Coyhaique and contribute to the supply of biomass for use in energy.

Key words: Salix, Energy, Grow, Diversification, Biomass.

\section{INTRODUCCIÓN}

Ampliar y diversificar la matriz energética de Chile, promoviendo la generación de energía a partir de fuentes renovables no convencionales es uno de los objetivos estratégicos planteados por el Ministerio de Agricultura de Chile y acogido por el Instituto Forestal.

Entendiendo biomasa como toda materia orgánica que proviene de árboles, plantas y desechos de animales que pueden ser convertidos en energía. La biomasa, particularmente de origen forestal, ha 
permitido el desarrollo acelerado de tecnologías de recolección, transformación y combustión, estando ya en un nivel relativamente dominado.

En Chile la biomasa se emplea principalmente para la producción energía calórica (térmica) y energía eléctrica, ya sea mediante su uso como leña, astillas, pellet y en plantas de cogeneración que se abastecen de los residuos de los procesos industriales (licor negro, corteza, otros), consumiendo la energía que demanda el funcionamiento de su industria y vendiendo los excedentes al SIC (Sistema Interconectado Central), la red de distribución nacional.

Actualmente en el país existen diversas fuentes de biomasa leñosa para la generación de energía, principalmente proveniente del manejo y subproductos de la silvicultura (raleos, cosecha) así como también subproductos de la industria del aserrío y celulosa.

Un componente abordado en la investigación tecnológica forestal orientada a la búsqueda de alternativas energéticas en Chile, corresponde a la identificación de especies forestales que pueden ser utilizadas en generación de biomasa para energía, incluyendo en ello a las plantaciones Bioenergéticas. Estas plantaciones se han considerado las más eficientes para el abastecimiento de plantas generadoras de energía a partir de biomasa y son ampliamente usadas en España, Estados Unidos, Inglaterra, Suecia, Nueva Zelanda, Alemania y Brasil para la generación de energía térmica y eléctrica. Su característica principal es que se establecen con especies forestales de rápido crecimiento y corta rotación.

Existen antecedentes acerca de las atractivas propiedades como combustible de Acacia, Eucalyptus y Salix, los cuales INFOR quiere validar según su adaptación y productividad como plantaciones dendro energéticas en diferentes territorios en el país.

Estas plantaciones presentan densidades variables con rotaciones estimadas de 2 a 4 años. Se caracterizan por requerir medidas de silvicultura intensiva, control de competencia y fertilización. Un aspecto fundamental de esta propuesta en el país, es que deben establecerse en suelos no utilizables por la agricultura.

Otra premisa a considerar es la identificación de la especie más adecuada para cada sitio, los modelos de manejo más apropiados y las herramientas de gestión que apoyen el proceso. En este escenario se requiere de:

- Cuantificar y caracterizar los suelos forestales disponibles para establecer plantaciones dendroenergéticas,

- Utilizar e introducir al país especies dendroenergéticas de interés, como Acacias (dealbata, melanoxylon, mearnsii, saligna (IV Región)), Eucalipto (nitens, camaldulensis, cladocalyx, sideroxylon), y especies o variedades de Salix, entre otras.

- Identificar las mejores especies para los distintos sitios,

- Desarrollar modelos de gestión adecuados

- Caracterizar la biomasa obtenida y su productividad

- Evaluar aspectos de manejo y cosecha,

- Generar antecedentes de modelos de abastecimiento en coordinación con los demandantes del recurso dendroenergético, entre otros.

Adicionalmente, se requiere de una caracterización energética que debe considerar los siguientes análisis:

- \% de Humedad,

- Poder Calorífico

- \% de cenizas

- Densidades

- Requerimientos a cumplir (procesos y legislación).

La bioenergía representa, además, una valiosa alternativa para la mitigación del cambio climático global, siendo neutra en las emisiones de carbono frente a las importantes emisiones que generan combustibles como el petróleo, sus derivados y el carbón mineral, siendo una energía renovable y limpia.

Para acelerar la incorporación a la producción de especies dendroenergéticas es aconsejable estudiar a nivel nacional aquellas alternativas conocidas y probadas en el mundo. Por otra parte, también es válido 
prospectar entre los recursos locales las especies con probada adaptación a las condiciones de sitio imperantes en el país, e identificar aquellas que presenten características compatibles con la producción de bioenergía.

Al respecto, las especies de Salix, son una interesante alternativa de producción dendroenergética en sitios específicos, ya que presentan un rápido crecimiento, que permitiría obtener biomasa en rotaciones cortas (3 a 5 años), y son especies que se adaptan a distintas situaciones edafoclimáticas en el país (Pinilla y Navarrete, 2010).

Junto con estudiar especies potenciales, es necesario conocer antecedentes acerca de su propagación, establecimiento, silvicultura, utilización y mejoramiento genético, entre otros. Este se correlaciona con los estudios de adaptación y rendimiento bajo diferentes sitios.

Este trabajo, actualmente en desarrollo, permite generar información focalizada en el fomento y utilización de la biomasa forestal para la generación de energía renovables, con el objetivo de apoyar el desarrollo; incrementar la productividad de los bosques en el país; diversificar la matriz energética nacional; y aportando, además, a la construcción de políticas públicas y el emprendimiento silvícola y tecnológico requeridos para los diferentes sectores involucrados.

En la Región de Aysén no existen antecedentes acerca de especies para su uso en energía, a excepción de algunos estudios realizados por INIA e INFOR con especies de álamos y sauces. Se requieren mayores antecedentes de las especies y de su manejo para el abastecimiento seguro de unidades de generación que requieran de biomasa para su funcionamiento.

En la búsqueda de opciones regionales para el suministro de biomasa para energía, se han iniciado estudios sobre las características de especies de Salix establecidas en el sector de Viviana Norte, comuna de Puerto Aysén, Región de Aysén, para obtener antecedentes de productividad y poder calorífico.

La introducción de híbridos y cultivares de Salix involucra ventajas económicas que mejoran la rentabilidad del negocio forestal como consecuencia de su rápido crecimiento y corta rotación. Además, incorporan ventajas sociales al brindar nuevas alternativas a los pequeños y medianos propietarios. Lo anterior supone también ventajas ambientales debido a la función de protección de los árboles a suelos fluviales, inundados y erosionados, y a su aporte a la mitigación de problemas de contaminación (Riquelme et al., 2019).

Atendiendo al interés y potencialidad de las especies del género Salix (sauces) para la Región de Aysén, en este artículo se da cuenta de los resultados obtenidos en la evaluación de un ensayo donde se prueban distintas especies de Salix. Esta unidad, establecida en las cercanías de Coyhaique camino a Puerto Aysén fue medida y sus datos fueron sometidos a análisis para caracterizar el crecimiento y potencial energético de las especies. El objetivo es caracterizar la respuesta de dos clones de Salix mediante variables como, la supervivencia, altura, diámetros e indicadores de productividad y capacidad para su uso en energía.

\section{MATERIAL Y MÉTODO}

\section{Área de Estudio}

Se evaluó una experiencia de plantación de sauces correspondiente a una cortina cortaviento de $290 \mathrm{~m}$ de largo, establecida en el año 2013 con fines dendroenergéticos en el sector de Viviana Norte (VN), a 45

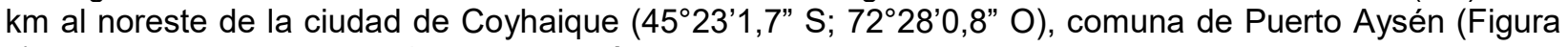
1). La cortina tiene un diseño de plantación en tres bolillos y un espaciamiento de $0,9 \mathrm{~m}$ entre hilera y 1,0 m sobre la hilera, en donde se estableció a modo de zona Buffer dos hileras con especies de sauce. 


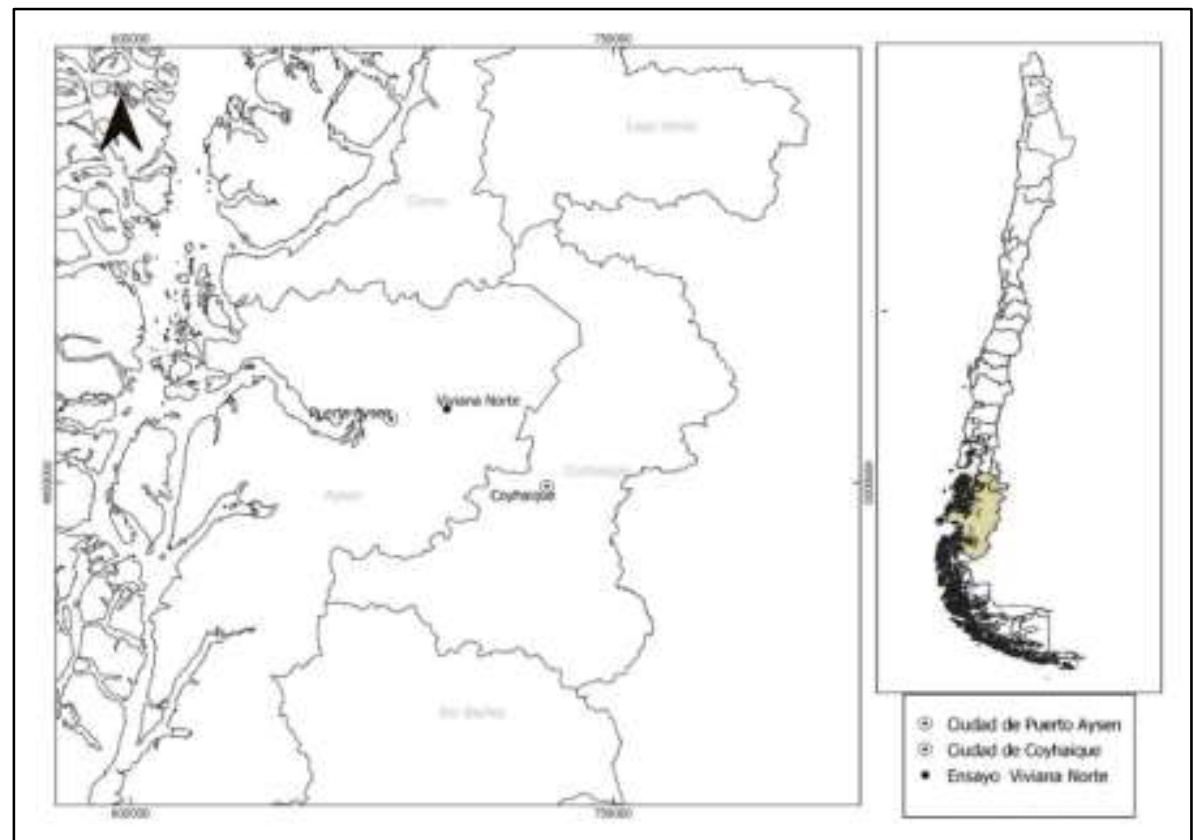

Figura 1. Ubicación sector del estudio

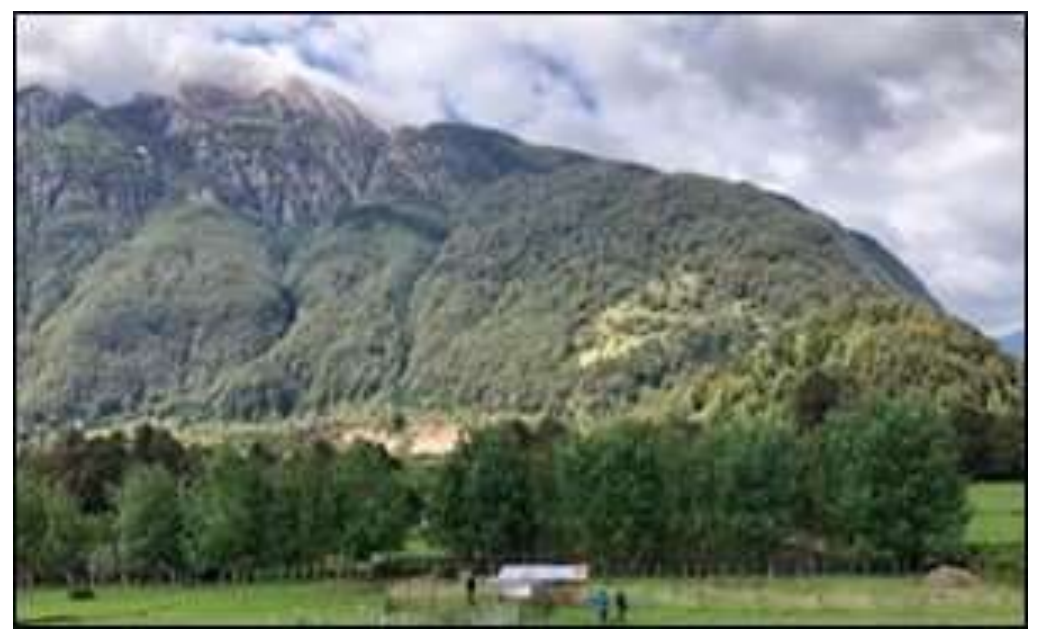

Figura 2. Vista sector con sauces seleccionados para el estudio

Según Riquelme et al. (2019) el sector presenta una vegetación predominante de bosque Siempreverde, y está dentro de la denominada Zona Húmeda de Aysén, con $2.640 \mathrm{~mm}$ anuales de precipitación (promedio 1960 - 2013). La temperatura media anual de la misma localidad es de $9^{\circ} \mathrm{C}$, mientras que la máxima media llega a $12,8^{\circ} \mathrm{C}$ y la mínima media a $6^{\circ} \mathrm{C}$. El mes más cálido (enero) presenta una temperatura media de $13,6^{\circ} \mathrm{C}$, con una máxima media de $17,9^{\circ} \mathrm{C}$, y una mínima media de $6^{\circ} \mathrm{C}$. El mes más frío (julio) tiene una temperatura media de $3,8^{\circ} \mathrm{C}$, con una máxima media de $6,8^{\circ} \mathrm{C}$ y una mínima media de $1,6^{\circ} \mathrm{C}$. La amplitud térmica media entre el mes más cálido y el más frío es de $9,8^{\circ} \mathrm{C}$ (Hepp y Stolpe, 2014).

El suelo es de drenaje imperfecto, textura franca, profundidades de hasta $88 \mathrm{~cm}$ y material parental de cenizas volcánicas (Stolpe y Hepp, 2014). 


\section{Especies utilizadas}

-Salix alba

Árbol de hasta 25 metros de altura; con hojas aserradas de color gris plateado, envés sedoso y tamaño aproximado de $5 \times 12 \mathrm{~cm}$. Sus flores tienen amentos en forma cilíndrica, que crecen en primavera. La corteza es de color grisáceo. Su madera es flexible y liviana; se utiliza para hacer cerillas, entre otros utensilios. Es especialmente adecuada para sitios con presencia de agua.

Las estacas de esta especie fueron obtenidas desde la zona de Coelemu, Región del Biobío.

\section{-Salix cinérea}

Arbusto de tamaño mediano con ramas gruesas; ocasionalmente árbol de $6 \mathrm{~m}$ de altura. Ramas jóvenes de color pardo grisáceo o pardo oscuro. Madera de las ramas de 2-4 años con numerosas costillas longitudinales, de hasta $2 \mathrm{~cm}$ de largo y $0,5 \mathrm{~mm}$ de altura. Hojas de hasta $12 \mathrm{~cm}$ de largo y $4,5 \mathrm{~cm}$ de ancho, ovadas u oblanceoladas, más anchas por encima de la mitad, inicialmente con pilosidad corta y tomento gris por ambas caras, más tarde glabras, de color verde grisáceo o verde oliváceo pardusco por el haz, verde azulado o verde grisáceo por el envés

Es una planta útil para consolidar suelos sueltos, taludes, bancos, taludes y para la reforestación de áreas húmedas no cultivadas. Debido a sus características es ideal en espacios pequeños, con presencia de agua.

Sus ramas se pueden usar para construir cabañas de caza también llamadas cestas. La corteza proporciona taninos y salicina. De esta última se obtiene el ácido salicílico, con acción tónica, antirreumática, febrífuga y astringente. Las hojas de los sauces actúan como cicatrizantes, astringentes y sedantes ${ }^{1}$.

Las estacas de esta especie fueron obtenidas desde la zona de Valdivia, Región de Los Ríos.

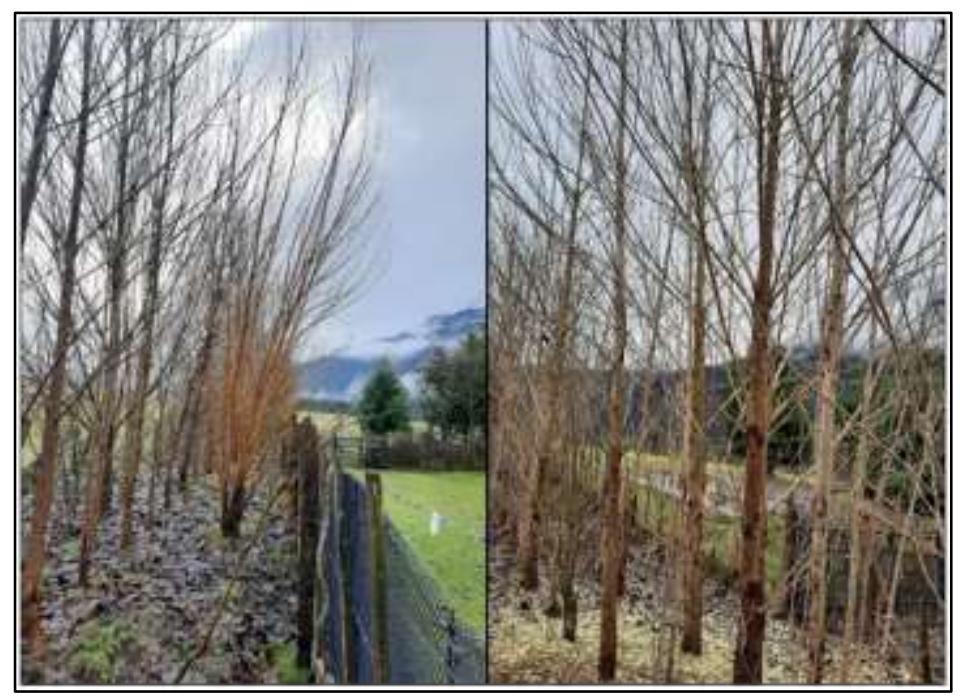

Figura 3. Sauces seleccionados para el estudio

\section{Evaluaciones realizadas}

La medición evaluada corresponde a la efectuada después de 8 años de establecidos los individuos en terreno.

${ }^{1}$ http://antropocene.it/es/2019/02/25/salix-cinerea/ 
Para la caracterización del crecimiento se consideraron 5 árboles por variedad clonal, según distribución diamétrica. A partir de ellos se estimó los parámetros dasométricos, índice de productividad (DAP ${ }^{2 *}$ altura) y antecedentes de peso verde.

La medición de los clones consideró el registro de las variables altura $(\mathrm{H})$, diámetro de cuello (DAC) y número de vástagos por planta (NV). Las variables dasométricas $\mathrm{H}$ y $\mathrm{DAC}$ se midieron para cada vástago de las plantas evaluadas.

Luego de la medición se voltearon los árboles seleccionados, se trozaron y desrramaron, de tal forma de poder pesarlos en terreno, obteniendo el dato del peso verde total de los individuos con una balanza (Figura 4).

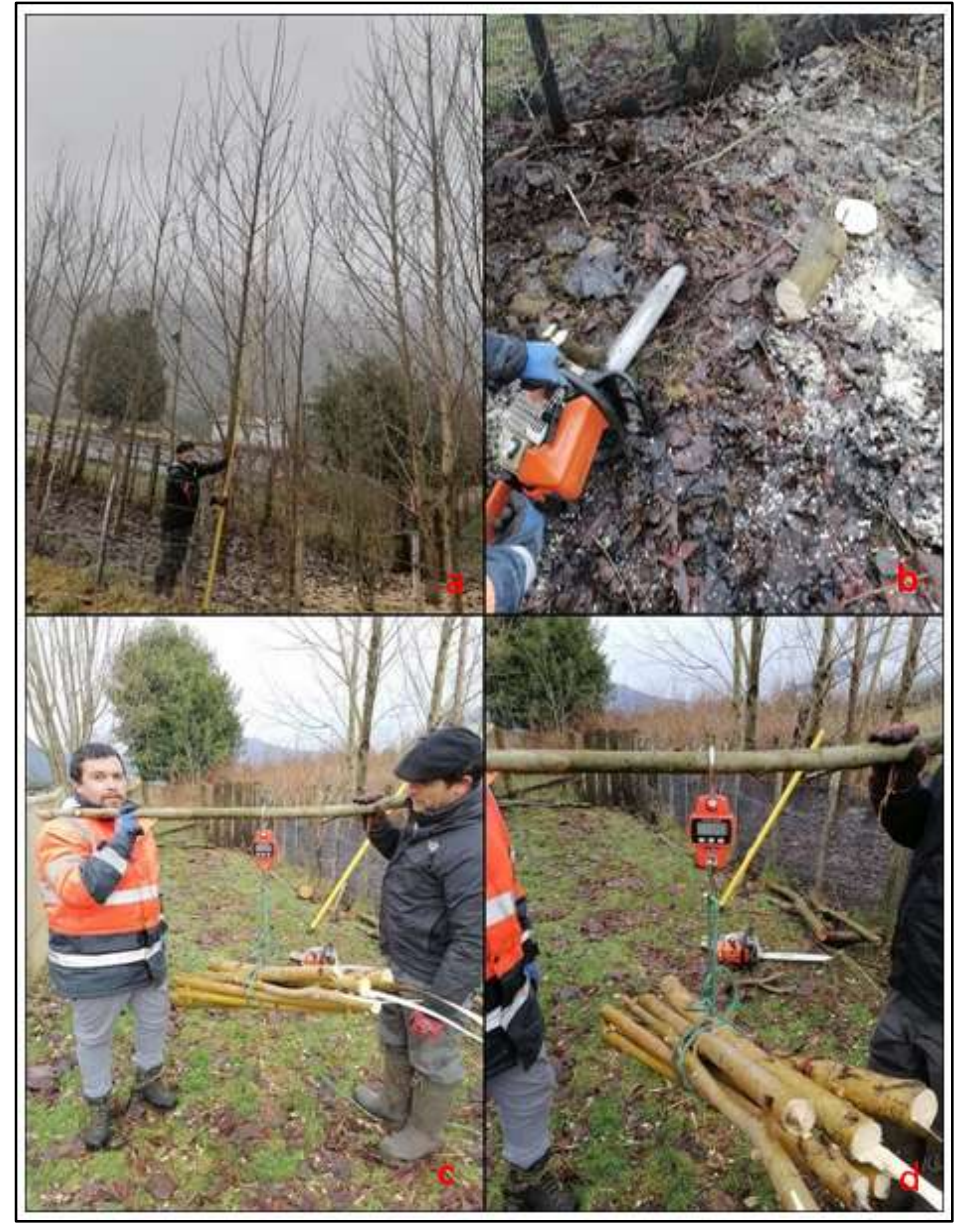

Figura 4. Medición de altura de los sauces en la cortina cortavientos (a); Volteo de los sauces seleccionados (b); Pesaje de biomasa verde y registro de datos (c y d).

Posteriormente, para la estimación de la biomasa seca, se etiquetó y empaquetó el material según componente, árbol y especie para su traslado a dependencias de INFOR en Coyhaique, donde fueron dispuestas en un secador convencional a $103^{\circ} \mathrm{C}$ por 15 a 17 horas para bajar su contenido de humedad hasta obtener peso constante (figura 5).

\section{Caracterización Biomasa}

Muestras de biomasa de las dos especies de Salix, fueron enviadas a laboratorio para su caracterización de acuerdo a la Norma ISO 17225-2 Biocombustibles sólidos, Especificaciones de combustibles y clases. Estas evaluaciones fueron efectuadas usando muestras de las mismas especies pero obtenidas el año anterior desde un banco clonal administrado por INFOR Coyhaique. Los análisis correspondieron a: 
- Contenido de humedad

- Contenido de ceniza

- Poder Calorífico Superior e Inferior

- Análisis elemental (Carbono, Hidrógeno, Nitrógeno y Azufre

- Determinación de Cloro

Los Análisis Elementales se efectuaron en Laboratorio de la Universidad de Concepción con un equipo Analizador Elemental marca Leco CHN 628 y 628 S, siguiendo protocolo del manual del equipo para medición en muestras sólidas y utilizando curva de calibración realizada con estándar EDTA para el módulo Carbono-Hidrógeno-Nitrógeno y Rice Flour para el módulo de Azufre².

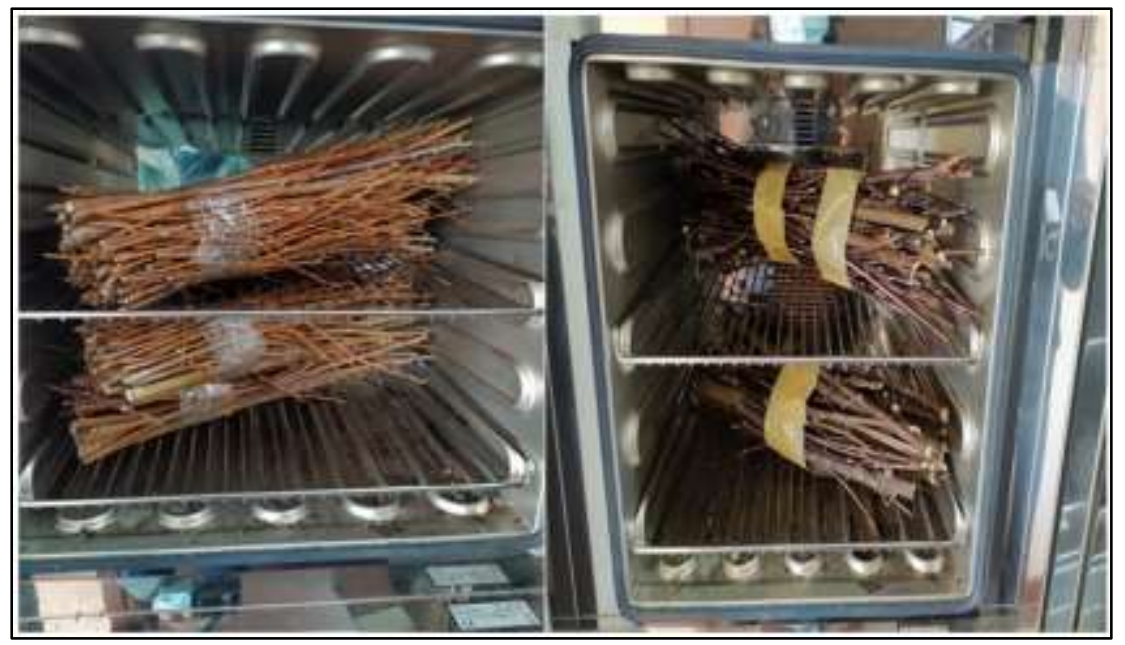

Figura 5. Secado en paquetes de clones de Salix cinerea y Salix alba

\section{RESULTADOS}

Los resultados de las mediciones de los árboles y del pesaje en verde de los componentes de la biomasa aéreas se presentan en el Cuadro 1.

Cuadro 1. Resultados de sauces seleccionados y biomasa verde obtenida (8 años)

\begin{tabular}{|c|c|c|c|c|c|c|c|c|c|c|c|}
\hline \multirow[b]{2}{*}{ Especie } & \multirow[b]{2}{*}{ ID } & \multirow[b]{2}{*}{$\begin{array}{l}\text { DAP } \\
(\mathbf{c m})\end{array}$} & & \multirow[b]{2}{*}{$\begin{array}{l}\text { Dac } \\
\text { (cm) }\end{array}$} & \multirow{2}{*}{$\begin{array}{l}\text { Altura } \\
(\mathrm{m})\end{array}$} & & \multirow{2}{*}{$\begin{array}{c}\mathbf{N}^{\circ} \\
\text { Fustes }\end{array}$} & \multirow{2}{*}{$\begin{array}{c}\text { Índice } \\
\text { Productividad } \\
\left(\mathrm{cm}^{3}\right)\end{array}$} & \multicolumn{3}{|c|}{ Peso verde $(\mathrm{Kg})$} \\
\hline & & & & & & & & & $\begin{array}{l}\text { ramas - } \\
\text { ramillas }\end{array}$ & $\begin{array}{l}\text { tronco } \\
\text { leñoso }\end{array}$ & $\begin{array}{c}\text { Total } \\
\text { individuo }\end{array}$ \\
\hline \multirow{6}{*}{$\begin{array}{l}\text { Salix } \\
\text { alba }\end{array}$} & $\mathrm{A} 1$ & 7,5 & & 14,5 & 6,94 & & 2 & 39.038 & 6,1 & 16,7 & 22,8 \\
\hline & A2 & 5,2 & & 9,3 & 8,75 & & 1 & 23.660 & 2,8 & 12,5 & 15,3 \\
\hline & A3 & 5,4 & & 15,5 & 6,71 & & 2 & 19.566 & 11,1 & 27,7 & 38,8 \\
\hline & A4 & 1,5 & & 4,5 & 5,07 & & 3 & 1.141 & 2,5 & 3,0 & 5,5 \\
\hline & A5 & 4,0 & & 11,0 & 6,18 & & 2 & 9.888 & 5,5 & 2,3 & 7,8 \\
\hline & Prom & 4,7 & $\mathbf{a}$ & 11,0 & 6,73 & $\mathbf{a}$ & 2 & 18.659 & 5,6 & 12,4 & 18,0 \\
\hline \multirow{6}{*}{$\begin{array}{c}\text { Salix } \\
\text { cinerea }\end{array}$} & C1 & 3,0 & & 4,5 & 5,95 & & 2 & 5.355 & 4,1 & 5,4 & 9,5 \\
\hline & $\mathrm{C} 2$ & 1,0 & & 5,0 & 2,93 & & 2 & 293 & 2,3 & 1,4 & 3,7 \\
\hline & C3 & 3,5 & & 6,0 & 5,93 & & 3 & 7.264 & 6,7 & 8,7 & 15,4 \\
\hline & C4 & 4,5 & & 8,5 & 7,14 & & 3 & 14.459 & 6,5 & 13,4 & 19,9 \\
\hline & C5 & 2,0 & & 3,0 & 5,70 & & 1 & 2.280 & 1,1 & 2,4 & 3,5 \\
\hline & Prom & 2,8 & $\mathbf{a}$ & 5,4 & 5,53 & $\mathbf{a}$ & 2 & 5.930 & 4,1 & 6,3 & 10,4 \\
\hline \multicolumn{2}{|c|}{ Promedio General } & 3,8 & & 8,2 & 6,13 & & 2 & 12.294 & 4,9 & 9,4 & 14,2 \\
\hline
\end{tabular}

Los resultados generales indican un mayor crecimiento de Salix alba, en altura dap e índice de productividad (Cuadro 1), no obstante esta diferencias entre especie no son estadísticamente significativas.

${ }^{2}$ Informe de Ensayo Caracterización Biomasa RT-007-156. Informe de proyecto 
La relación DAP-Altura por especie se presenta en la figura 6. En el gráfico se puede apreciar una relación estable y creciente entre el valor del DAP $(\mathrm{cm})$ y la altura $(\mathrm{m})$ de los árboles.

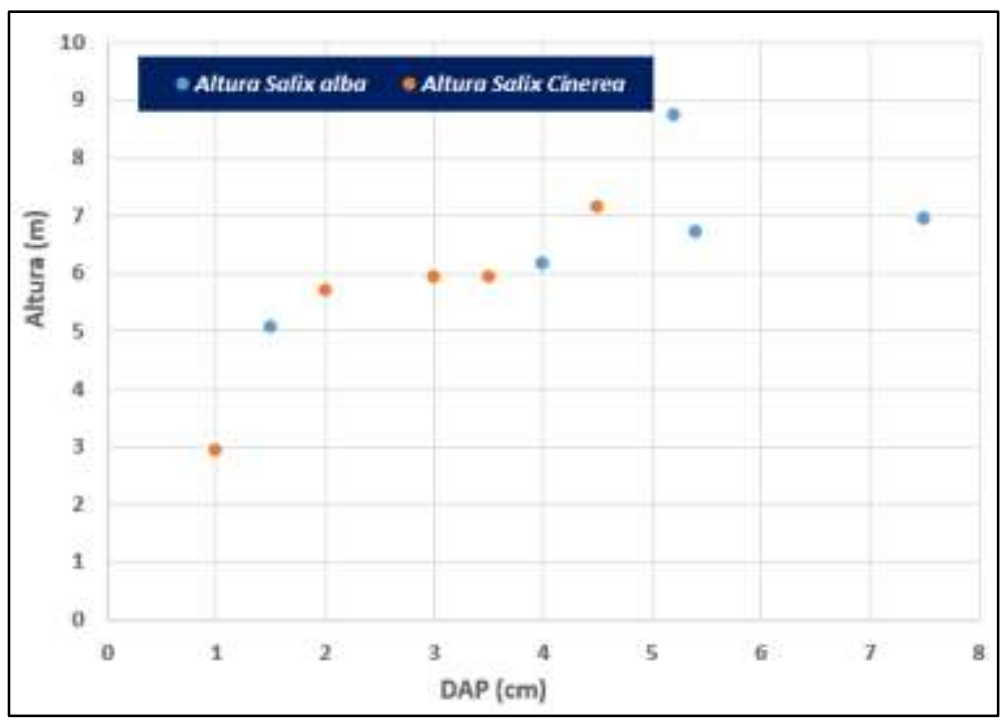

Figura 6. Relación DAP-Altura para Salix cinerea y S. alba a los 8 años de edad.

A partir de estos resultados se puede señalar que Salix alba presenta interesantes resultados, lo que sugiere que puede ser una especie para continuar su testeo en especial en zonas más húmedas como fuente de biomasa para energía en zonas similares a dónde está establecido.

\section{Peso Verde de Biomasa Aérea}

El mayor crecimiento en biomasa aérea lo presenta la variedad $S$. alba, con un peso verde promedio del árbol de 18 kilos, lo que llevado a la hectárea podría significar un total cercano a las 90 t/ha y una productividad media de 11,25 t/ha/año. Lo anterior asumiendo un espaciamiento medio de $1 \times 1$ metros y una ocupación del $50 \%$ del sitio debido a los espacios requerido para las actividades mecanizadas.

Los árboles de $S$. cinérea presentaron una biomasa aérea verde con 10,4 kilos, lo que con los mismos supuestos anteriores significaría un total de 52 t/ha y 6,5 t/ha/año de crecimiento medio.

La figura 7 presenta la relación general entre el DAP y el peso verde aéreo de los árboles de ambas especies. 


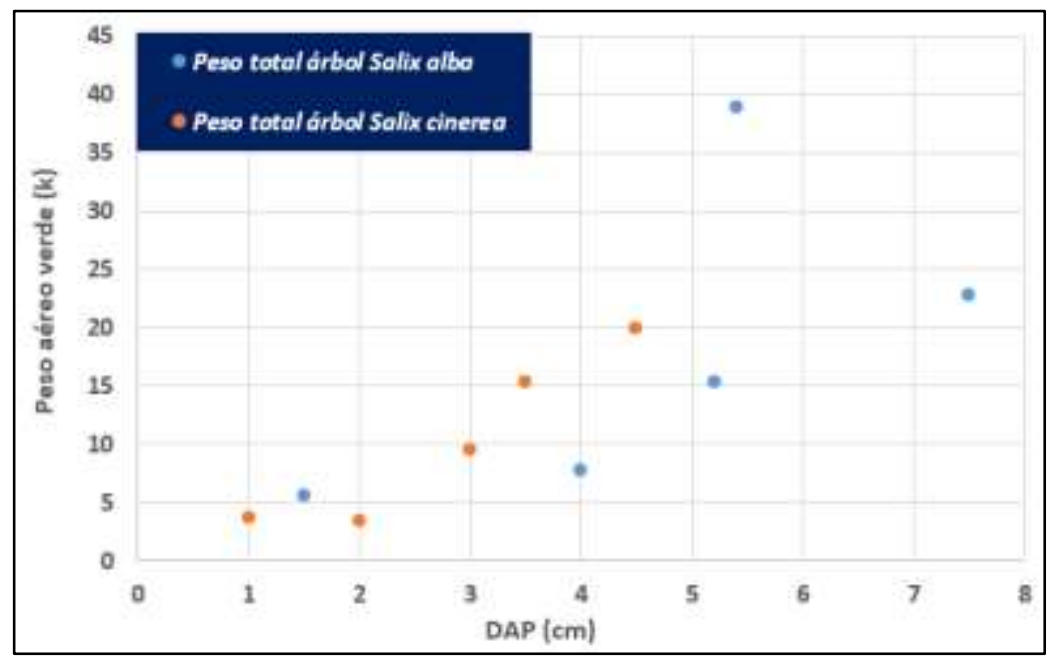

Figura 7. Relación DAP-Peso Verde en Salix cinerea y $S$. alba a los 8 años de edad.

La distribución porcentual de la biomasa total del árbol en los componentes ramillas, ramas y fuste se presentan en el Cuadro 2.

Cuadro 2. Distribución porcentual de biomasa de árboles de Salix cinerea y S. alba a los 8 años de edad.

\begin{tabular}{|c|c|c|c|c|c|}
\hline \multirow{3}{*}{ Especie } & \multicolumn{5}{|c|}{ Peso verde } \\
\hline & \multirow{2}{*}{$\begin{array}{c}\text { Total árbol } \\
(\mathrm{Kg})\end{array}$} & \multicolumn{2}{|c|}{ Ramas ramillas } & \multicolumn{2}{|c|}{ Tronco leñoso } \\
\hline & & $(\mathrm{Kg})$ & $(\%)$ & $(\mathrm{Kg})$ & $(\%)$ \\
\hline \multirow{5}{*}{$\begin{array}{l}\text { Salix } \\
\text { alba }\end{array}$} & 22,8 & 6,1 & 26,8 & 16,7 & 73,2 \\
\hline & 15,3 & 2,8 & 18,3 & 12,5 & 81,7 \\
\hline & 38,8 & 11,1 & 28,6 & 27,7 & 71,4 \\
\hline & 5,5 & 2,5 & 45,5 & 3,0 & 54,5 \\
\hline & 7,8 & 5,5 & 70,5 & 2,3 & 29,5 \\
\hline \multirow[t]{2}{*}{ Prom } & 18,0 & 5,6 & 37,9 & 12,4 & $62,1 \%$ \\
\hline & 9,5 & 4,1 & 43,2 & 5,4 & 56,8 \\
\hline Salix & 3,7 & 2,3 & 62,2 & 1,4 & 37,8 \\
\hline \multirow{3}{*}{ cinerea } & 15,4 & 6,7 & 43,5 & 8,7 & 56,5 \\
\hline & 19,9 & 6,5 & 32,7 & 13,4 & 67,3 \\
\hline & 3,5 & 1,1 & 31,4 & 2,4 & 68,6 \\
\hline Prom & 10,4 & 4,1 & 42,6 & 6,3 & $57,4 \%$ \\
\hline Promedio general & 14,22 & 4,87 & 34,2 & 9,35 & 65,8 \\
\hline
\end{tabular}

En el cuadro anterior se observa que en promedio un $34 \%$ del peso verde del árbol corresponde a ramas y ramillas, mientras que un $66 \%$ corresponde al fuste. En $S$. alba el $38 \%$ del peso verde aéreo corresponde a ramas o ramillas, mientras que el $62 \%$ corresponde al fuste. En el caso de Salix cinérea, aumenta la proporción de biomasa de ramas y ramillas a un $43 \%$, y se reduce la del fuste a $57 \%$.

La relación entre el dap de los árboles y la forma en que distribuyen su biomasa entre ramas-ramillas y fuste se grafica en la figura 8, observándose que a medida que aumenta el DAP, disminuye la componente de biomasa de ramas y ramillas y aumenta la participación de la biomasa correspondiente al fuste. 


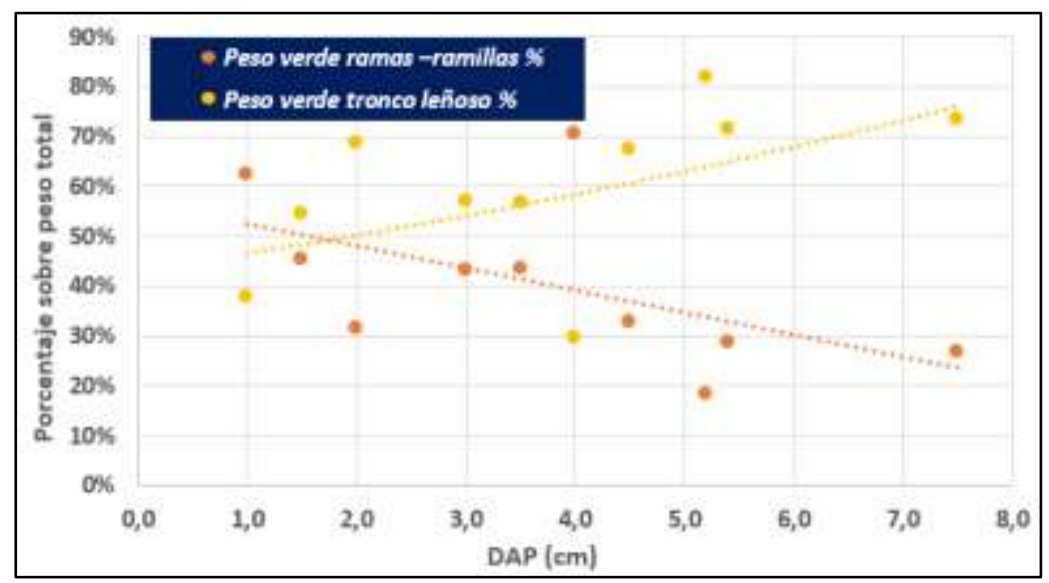

Figura 8. Distribución porcentual del peso verde de ramas-ramillas y fuste en función del diámetro de individuos de Salix cinerea y $S$. alba de 8 años de edad.

\section{Peso Seco de Biomasa Aérea}

En general el peso seco obtenido corresponde a un $41 \%$ del peso verde de la biomasa cosechada (Cuadro 3). En anteriores estudios con diferentes especies de Salix este porcentaje correspondió a aproximadamente un 55\% (Pinilla y Navarrete, 2010).

Cuadro 3. Peso seco de ramas y ramillas de Salix cinerea y $S$. alba a la edad de 8 años.

\begin{tabular}{ccccccc}
\hline ESPECIE & ID & $\begin{array}{c}\text { DAP } \\
(\mathrm{cm})\end{array}$ & $\begin{array}{c}\text { Altura } \\
(\mathrm{m})\end{array}$ & $\begin{array}{c}\text { Peso Verde } \\
(\mathrm{Kg})\end{array}$ & $\begin{array}{c}\text { Peso Seco } \\
(\mathrm{Kg})\end{array}$ & $\begin{array}{c}\text { Peso Seco } \\
(\%)\end{array}$ \\
\hline \multirow{5}{*}{$\begin{array}{c}\text { Salix } \\
\text { alba }\end{array}$} & $\mathrm{A} 1$ & 7,5 & 6,94 & 6,10 & 2,57 & $42 \%$ \\
\cline { 2 - 7 } & $\mathrm{A} 2$ & 5,2 & 8,75 & 2,80 & 1,10 & $39 \%$ \\
\cline { 2 - 7 } & $\mathrm{A} 3$ & 5,4 & 6,71 & 11,10 & 4,73 & $43 \%$ \\
\cline { 2 - 7 } & $\mathrm{A} 4$ & 1,5 & 5,07 & 2,50 & 0,94 & $38 \%$ \\
\cline { 2 - 7 } & $\mathrm{A} 5$ & 4,0 & 6,18 & 5,50 & 2,18 & $40 \%$ \\
\hline \multirow{5}{*}{$\begin{array}{c}\text { Salix } \\
\text { cinerea }\end{array}$} & $\mathrm{Prom}$ & 4,7 & 6,70 & 5,60 & 2,30 & $40 \%$ \\
\cline { 2 - 7 } & $\mathrm{C} 1$ & 3,0 & 5,95 & 4,10 & 1,92 & $47 \%$ \\
\cline { 2 - 7 } & $\mathrm{C} 3$ & 1,0 & 2,93 & 2,30 & 0,95 & $41 \%$ \\
\cline { 2 - 7 } & $\mathrm{C} 4$ & 4,5 & 5,93 & 6,70 & 2,91 & $43 \%$ \\
\cline { 2 - 7 } & $\mathrm{C} 5$ & 2,0 & 5,14 & 6,50 & 2,84 & $44 \%$ \\
\cline { 2 - 7 } & Prom & 2,8 & 5,50 & 4,10 & 0,38 & $34 \%$ \\
\hline \multicolumn{2}{c}{ Promedio General } & 3,8 & 6,13 & 4,87 & 2,05 & $41 \%$ \\
\hline
\end{tabular}

La biomasa aérea seca de ramas y ramillas de $S$. alba, correspondiente a un $40 \%$ de la biomasa verde inicial. En el caso de $S$. cinérea este factor llegó a un $42 \%$. Si esto se lleva a la hectárea utilizando los supuestos anteriores (espaciamiento medio de $1 \times 1$ metros y una ocupación del $50 \%$ del sitio), es posible estimar en $36 \mathrm{tMS} / \mathrm{ha}^{3}$, con un valor cercano a los $5 \mathrm{tMS} / \mathrm{ha} / \mathrm{año}$. En el caso de Salix cinérea ello representaría un valor de materia seca cercano a 22 t/ha y $3 \mathrm{tMS} / \mathrm{ha} / \mathrm{año}$ de crecimiento medio.

\section{Caracterización de la Biomasa}

Los resultados de caracterización de la biomasa de $S$. cinerea y $S$. alba se resumen en el Cuadro 4 sobre la base de la materia seca.

\footnotetext{
${ }^{3}$ tMS/ha, toneladas de materia seca por hectárea
} 
Cuadro 4. Caracterización de la biomasa de ramas y ramillas de Salix cinerea y $S$. alba a la edad de 7 años.

\begin{tabular}{lrr}
\hline \multicolumn{1}{c}{ Parámetro } & \multicolumn{2}{c}{ Especies } \\
\cline { 2 - 3 } & Salix alba & Salix Cinerea \\
\hline Contenido de Humedad (\%) & 50,38 & 51,47 \\
\hline Contenido en Cenizas $(\%)$ & 1,40 & 2,91 \\
\hline Poder Calorífico Superior $(\mathrm{J} / \mathrm{g})$ & 19.333 & 19.481 \\
\hline Poder Calorífico Inferior $(\mathrm{J} / \mathrm{g})$ & 18.009 & 18.163 \\
\hline Carbono $(\%)$ & 49,840 & 49,370 \\
\hline Hidrógeno (\%) & 6,075 & 6,054 \\
\hline Nitrógeno (\%) & 0,910 & 1,015 \\
\hline Azufre (\%) & 0,076 & 0,087 \\
\hline Cloro (\%) & 0,021 & 0,020 \\
\hline
\end{tabular}

\section{DISCUSIÓN}

Para acelerar la incorporación de especies forestales al abastecimiento de biomasa para energía, es necesario estudiar a nivel nacional y local aquellas alternativas conocidas y probadas en el mundo, especialmente cuando estas cuentan con los antecedentes técnicos que las avalan y recomiendan para este uso. Por otra parte, también es válido prospectar entre los recursos locales en busca de especies con probada adaptación a las condiciones de sitio imperantes en el país, y tratar de identificar entre ellas a algunas que presenten características compatibles con la producción de bioenergía.

En términos generales Salix alba mostró una adecuada adaptación a una zona de alta disponibilidad hídrica $(2.640 \mathrm{~mm})$ y exhibe mejor desarrollo que $S$. cinerea. Presentó además un fuste recto, características que favorece su manejo, cosecha y utilización como biomasa para energía. En general las salicáceas provienen de zonas con inviernos fríos, algo rigurosos, por lo cual las bajas temperaturas del sector donde fueron establecidas no constituyen un factor climático limitante (Leonardis, 1960).

Las menores exigencias de clima y suelo Salix alba, junto con su plasticidad, le permiten obtener a los 8 años diámetros de $4,7 \mathrm{~cm}$, alturas de $6,17 \mathrm{~m}$ e índices de productividad de $18.659 \mathrm{~cm}^{3}$. A nivel de árboles individuales esta especie registró valores de DAP de hasta $7,5 \mathrm{~cm}$ y altura de hasta $8,8 \mathrm{~m}$. Como antecedente de referencia, Riquelme et al. (2019) señalan que en la misma zona el clon de álamo Unal a los siete años de edad presentó un diámetro de $5 \mathrm{~cm}$, altura de 3 metros e Índices de Productividad de $8.311 \mathrm{~cm}^{3}$. En el mismo sentido Pinilla y Navarrete (2010) mencionan que Salix alba establecido en la zona de Cañete, Región del Biobío, presentó a los 3 años un DAC de 28,2 a 32,6 mm, una altura de 2,2 a 2,77 metros y un peso verde aéreo de $0,6 \mathrm{Kg}$; en el mismo sitio individuos de Salix cinérea registraron valores de 56 a $30 \mathrm{~mm}$ en DAC, 3,3 a 3,5 $\mathrm{m}$ de altura y 3,1 a $3,3 \mathrm{Kg}$ de peso verde aéreo.

El Poder Calorífico incide en la cantidad de biocombustible para producir una unidad de energía. De ahí la importancia de contar con especies cuya biomasa entregue un alto valor de esta variable. Un mayor poder calorífico genera evidentemente una biomasa de mayor calidad energética. Al respecto, los resultados obtenidos indican valores de 19.333 y de $19.481 \mathrm{~J} / \mathrm{g}$ para $S$. alba y $S$. cinérea, respectivamente. En términos de kilocalorías equivalen a 4,62 y $4, .65 \mathrm{kcal} / \mathrm{g}$. Como antecedente se señala que el poder calorífico determinado para maderas y cortezas de distintas procedencias de Salix varía entre 3,91 a 4,36 kcal/g (Durán, 1998), mientras que estudios realizados por INFOR con Salix humboldtiana indican 4,3 kcal/g (Pinilla y Navarrete, 2010). Otros antecedentes mencionados por Covacevich (1979) y Pinilla y Hernández (2010) para el poder calorífico superior de madera seca en horno de Pinus radiata, Acacia caven, A. delbata y Eucalyptus globulus entregan valores de 4,23 a 4,82 $\mathrm{kcal} / \mathrm{g}$.

Los resultados confirman la aptitud de las especies evaluadas para su uso en la generación de energía y son similares a lo reportado en otros países, tanto en materia seca como en poder calorífico, lo que permitiría generar una línea de investigación en torno a la silvicultura y utilización de Salix para el abastecimiento de biomasa para energía, en sitios adecuados para el desarrollo de estas especies, y especialmente en la zona de Coyhaique, de modo de participar de las respuestas para los desafíos que presenta la zona para la calefacción y generación de energía.

A partir de estos resultados Salix alba sería una especie adecuada para sectores con condiciones similares a Viviana Norte (Zona Húmeda de Aysén). A lo anterior se agrega la capacidad de rebrotar 
desde tocón, lo que, unido a su rápido desarrollo, adaptabilidad y simple silvicultura, hace que Salix presente un alto potencial para su uso en energía en la Región de Aysén.

Especies de Salix se emplean en plantaciones comerciales de alto rendimiento, manejadas en monte bajo, principalmente en suelos agrícolas y húmedos, para el abastecimiento de biomasa para centrales energéticas locales de producción combinada de energía térmica y eléctrica. En tales plantaciones el cultivo del sauce está mecanizado desde la plantación hasta la cosecha. Pinilla et al. (2015) señalan que la producción aproximada de biomasa de sauce cultivado comercialmente en Suecia es de 6 a 12 t/ha/año, según las condiciones del terreno, agregando que, en Chile, con S. viminalis para producción de mimbre para cestería (Región de O’Higgins) se han obtenido rendimientos de hasta 12 tMS/ha/año.

La evaluación realizada en este estudio se considera adecuada para los objetivos de la investigación, ya que se trata de individuos jóvenes, en una edad que se estima indicada para una rotación con objetivos dendroenergéticos. Por otra parte, los valores registrados desde las especies de Salix corresponden a situaciones sin manejo ni mejora genética, por lo que la información constituye una línea base para ganancias a generar en volumen o biomasa mediante silvicultura, manejo y el uso del mejoramiento genético.

Los resultados también permitirán caracterizar la calidad de la biomasa generada en relación a su crecimiento según sitio, edad, manejo y atributos para su uso en energía. Conocer el crecimiento en diferentes condiciones, permitirá establecer esquemas silviculturales que permitan la producción sustentable de biomasa.

Todos estos resultados son iniciales y fundamentales para efectuar una selección más certera de las especies, árboles y esquemas silvícolas con mejor desempeño para producción de biomasa para energía en la Región de Aysén. Aún no es posible generar información sobre el desempeño de la especie en rodales uniformes, sin embargo, es factible estimar interesantes perspectivas para su uso en plantaciones, con regímenes de manejo intensivo en monte alto y posteriormente en monte bajo, con distintos períodos de rotación y con diferentes fines productivos, investigación que INFOR se propone iniciar en el corto plazo.

\section{CONCLUSIONES}

Se requiere estudiar e incorporar especies resistentes a las condiciones de sitio de Coyhaique, para diversificar la oferta regional de biomasa para energía, ya sea térmica, sanitaria o para electricidad. Este aspecto es especialmente relevante debido a la alta dependencia de la leña como fuente de calefacción, por la presión del bosque nativo para conseguir leña, por la creciente complejidad en el abastecimiento y por los problemas de polución ambiental derivados del mal uso de la misma.

Después de ocho años de crecimiento Salix alba, tanto en variables del árbol como en biomasa aérea, Salix alba alcanzó un DAP de 4,7 cm y una altura de 6,7 m, mientras que $S$. cinerea registró valores de $2,8 \mathrm{~cm}$ y $5,5 \mathrm{~m}$ para DAP y altura, respectivamente. En términos de biomasa aérea verde, $S$. alba presentó un valor medio de $18 \mathrm{Kg}$, mientras que $S$. cinérea registró un peso verde de 10,4 $\mathrm{Kg}$; en promedio el $41 \%$ de esos valores corresponde a peso seco. En cuanto al Poder Calorífico Inferior, se obtuvo un resultado de 18.009 y $18.163 \mathrm{~J} / \mathrm{g}$ para Salix alba y Salix cinérea, respectivamente, y valores del Análisis Elemental que permiten su utilización como materia prima para energía.

La adaptación y crecimiento de estas especies en las condiciones de suelo y clima del sector en estudio entrega un primer antecedente para la región, como un material adecuado y promisorio de testear en mayor profundidad.

La selección de una especie para su uso en energía permite generar escenarios con ventajas económicas que aporten a la rentabilidad del negocio forestal, derivado de su crecimiento en cortas rotaciones. Esto representa, además, ventajas sociales y ambientales para pequeños y medianos propietarios, los que podrían utilizar estos resultados para el establecimiento de bosques con diferentes objetivos, incluido el de abastecimiento de biomasa para energía.

Se requiere de ampliar este tipo de estudios en la zona de Coyhaique de modo de incrementar las alternativas de oferta de biomasa para su uso en energía y con ello, la selección de especies con mejor desempeño para producción de biomasa. 


\section{AGRADECIMIENTOS}

Los autores agradecen a los señores Jorge Bustamante (Investigador) y Exequiel Díaz (Técnico) de la sede Coyhaique de INFOR, por su apoyo y colaboración para la realización de esta investigación.

\section{REFERENCIAS}

Covacevich, R. (1979). Poder Calorífico de Pino Insigne y de otras especies forestales chilenas. Universidad de Chile. Santiago.

Durán, C. (1998). Caracterización de Salix viminalis acorde a su contenido de extraíbles y capacidad energética total. Editorial Universidad de Concepción. Facultad de Ciencias Forestales

Hepp, C. \& Stolpe, N.B. (2014). Caracterización y propiedades de los suelos de la Patagonia Occidental (Aysén). Instituto Nacional de Investigaciones Agropecuarias, Centro de Investigación INIA Tamel Aike, Coyhaique, Chile.160 p.

Leonardis, R. (1960). Silvicultura de las Salicáceas. Implantaciones de bosques comerciales. INTA. Pp: 193-206.

Pinilla, J.C. \& Hernández, G. (2010). Poder calorífico de Acacia dealbata Link crecida en Chile. Ciencia \& Investigación Forestal, 16(3): 353-379. https://doi.org/10.52904/0718-4646.2010.354

Pinilla, J.C. \& Navarrete, M. (2010). Informe Técnico Proyecto 1: Desarrollo productivo de los bosques, de la industria forestal y fomento del uso de la madera. Promoción del uso dendroenergético de los productos forestales madereros, el caso de Acacia dealbata y Salix sp. Informe de proyecto Ministerio de Agricultura de Chile. Instituto Forestal, Sede Biobío. Concepción, Chile. 50 p

Pinilla, J.C., Chung, P. \& Navarrete, M. (2015). El Sauce chileno (Salix humboldtiana Willd) en la región del Biobío y su uso potencial en bosques plantados. INFOR, Informe Técnico №202. Santiago. 44 p. https://doi.org/10.52904/20.500.12220/20998

Riquelme, F., Salinas, J., Gutiérrez, B. \& Pinilla, J.C. (2019). Evaluación de ensayos de introducción de álamos en dos zonas Agroclimaticas de la Región de Aysén. Ciencia \& Investigación Forestal, 25(3). 7-22. https://doi.org/10.52904/0718-4646.2019.518.

Stolpe, N.B. \& Hepp, C. (2014). Caracterización taxonómica de los suelos de los valles de interés agropecuario de la región de Aysén (Patagonia Occidental-Chile). Institutito Nacional de Investigaciones Agropecuarias, Centro de Investigación INIA Tamel Aike, Coyhaique, Aysén-Patagonia, Chile. 168 p. 